\title{
Night of Darkness Campaign: Make Light Pollution Something Everybody Cares About
}

\author{
Friedel Pas \\ European Liaison Officer \\ International Dark-Sky Association, Aarschotsebaan 29, B-3191, Hever, Belgium \\ email: europe@darksky.org
}

\begin{abstract}
To battle light pollution issues, influence the market so that customers request only good lighting. An important factor in doing this is to convince decision makers that light pollution is something the whole society cares about. This article gives an overview of an existing campaign that does this and emphasizes some important factors to consider in your campaign.
\end{abstract}

Keywords. education and public outreach, light pollution, citizen-science campaign

Awareness campaigns have a common goal. Increase awareness with the public, but also convince politicians to take action - especially when campaigning against light pollution.

To maximize the impact of your campaign amongst politicians, it is important to increase your critical mass. Campaigns against light pollution are mostly done from the point of view of astronomy. Light pollution is an important issue to amateur and professional astronomers, but astronomers are small in number. Their reasons for preserving a dark night sky give the public the impression the attention is to a selective hobby or a profession done on far-away mountaintops - not affecting them.

But the impact of light pollution affects much more than astronomy. It impacts the environment, human health, energy consumption, wildlife and our culture. To increase your critical mass for your campaign, it is important that you involve organizations representing these areas. It is important to involve a broad range of interests to demonstrate that light pollution is something that affects everybody.

The more people participate in an event, the more you increase the critical mass of your campaign. The night of Darkness is an example of such a campaign that offers the public the opportunity to participate. The annual event take place on October 20, 2012 for the 17th time in Flanders, Belgium and has expanded to other regions in Europe. On that night, municipalities are requested to switch off their unnecessary lighting. About $2 / 3$ of the municipalities are participating in some way - including switching off monument lighting, reducing the number of illuminated light poles and turning off lighting in a region or sometimes a whole municipality or city. Local activities are being organized: star gazing parties, night nature promenades, storytelling and concerts in the dark. Local municipalities, astronomy clubs, environmental organizations and cultural organizations are collaborating to organize these events. In 2011, the campaign events attracted more than 26,000 participants.

At the same time, the events are unique in providing astronomy outreach to people who do not typically experience astronomy. With astronomy events, you attract participants that are already interested in astronomy. With an event that is a combination of different interests, you can present the beauty of the sky to people who have a variety of reasons for being at the event. Apart from raising awareness to general public and politicians, providing further education in light pollution prevention is also an important aspect of a successful campaign. 